\title{
Um relato de experiência de capacitação para a criação de jogos sérios em cursos de computação com foco em projetos reais
}

\author{
Abner Cardoso da Silva, Universidade Federal do Pará, abner.cardo@ gmail.com \\ Jefferson Pantoja, Universidade Federal do Pará, jffkd35@gmail.com \\ Jefferson Luís Ribeiro, Universidade Federal do Pará, jeffersonluiseng@gmail.com \\ Gilberto Nerino de Souza Junior, Universidade Federal do Pará, gilbertonerinojr@ gmail.com \\ Antonio Fernando Lavareda Jacob Junior, Universidade Estadual do \\ Maranhão,jacobjr@engcomp.uema.br \\ Ádamo Lima de Santana, Universidade Federal do Pará, adamo@ufpa.br
}

Resumo. O processo de ensino-aprendizagem em computação tende a ser mais eficaz com a realização de atividades práticas a partir do desenvolvimento de projetos que tratem de problemas reais. Nesse contexto o processo de implementação de jogos sérios pode auxiliar no aprendizado de novas tecnologias e aplicação de métodos dinâmicos. Este trabalho relata a experiência de um processo de capacitação para o desenvolvimento de jogos sérios envolvendo graduandos de cursos de computação. A aplicação de questionários, pré e pós-testes, permitiu estimar o interesse dos alunos e avaliar o nível de conhecimento no processo de ensino. Os alunos da capacitação geraram três protótipos com os seguintes propósitos: 1) educação da saúde bucal de adolescentes; 2) identificação de daltonismo em crianças; 3) desenvolvimento do equilíbrio em pessoas da terceira idade. Palavras chaves: capacitação, jogos sérios, aprendizagem ativa.

Abstract. The process of computer-assisted teaching tends to be more efficient with practical activities that address real life problems. In this context, serious games allow the learning and application of new technologies and dynamic procedures. This work details the capacitation of undergraduate students for the development of serious games with that purpose. Pre and post-test surveys allowed to estimate the students' interest and evaluation of the teaching methodology. Students involved with this work were able to develop three prototypes with the following purposes: 1) buccal health awareness in adolescents; 2) diagnosing color blindness in children; and 3) improvement of balance and steadiness in senior people.

Keywords: Capacitate, Serious games, Active learning

\section{Introdução}

Cada vez mais, crianças, jovens e adultos vêm se habituando a manipular os mais variados tipos de dispositivos computacionais. Este fato tem facilitado a aceitação de métodos de aprendizagem tornando-as mais atraentes para o usuário final. (Girard et al. 2013). Estas facilidades proporcionam uma maior disseminação dos jogos sérios (serious games), como ferramentas de disseminação de conhecimento.

Os jogos sérios podem ser definidos como simulações de processos reais desenvolvidos com o propósito de resolver um determinado problema ou melhorar o desempenho do utilizador (Vilela e Borges, 2013). O uso desta tecnologia tem como propósito tratar problemas reais com foco no ensino e conscientização de algum proveito (Kapp, 2012).

Os benefícios do uso de jogos sérios incluem a melhoria de pensamento estratégico, melhoria de habilidades psicomotoras, desenvolvimento de habilidades analíticas e habilidades computacionais (Savi e Ulbricht, 2008). Sendo assim, os jogos sérios não são centrados somente no entretenimento, mas também servem como auxiliares no processo de 
aprendizagem em áreas como: saúde, reabilitação, meio ambiente, educação, publicidade, etc. (Connolly et al. 2012).

Contudo, o processo de desenvolvimento de jogos deve ser tratado de forma não trivial, já que a implementação de um jogo digital faz uso de variadas áreas de conhecimento, como: programação, modelos de desenvolvimento de software, artes gráficas, sonoplastia, game design, animação, entre outros (Rodrigues et al. 2010). Somado a este obstáculo estão os quesitos inerentes aos jogos sérios que envolvem sempre uma área de atuação, geralmente, aplicada a um domínio de aplicação. Além disso, observa-se que a inserção do processo de ensino e desenvolvimento de jogos em cursos de graduação ainda não tem sido muito explorado (Carvalho et al. 2015).

Como meio de reduzir estas limitações, foi proposto um projeto chamado de Fábrica de Games. Este projeto é focado no ensino e capacitação de desenvolvimento de jogos e pretende favorecer o aprendizado de alunos dos cursos de computação por meio da integração entre professores e alunos na execução de projetos reais. Para alcançar este objetivo, utiliza-se técnicas da Aprendizagem Ativa para a realização de atividades interdisciplinares (Norman e Spohrer, 1996). Neste sentido, o presente trabalho constituiuse de um relato de experiência da capacitação realizada no primeiro semestre de 2015, a qual contou com 12 alunos dos cursos de computação da Universidade Federal do Pará.

\section{Trabalhos Correlatos}

Schmidtet al. (2011) descreve uma estratégia de aprendizado ativa denominada PBL (Problem Based Learning), que se baseia em repassar uma série de problemas, pertinentes de um determinado tema à um grupo de aprendizes. Estas atividades devem ser solucionadas a partir de conhecimentos obtidos em pesquisas autônomas e com o auxílio de um tutor responsável por introduzir o embasamento teórico além de coordenar os métodos e modelos de resolução optados pelos alunos. São citadas como características principais da metodologia baseada em PBL: (i) problemas são usados como estopim para o aprendizado; (ii) estudantes são organizados em pequenos grupos; (iii) o aprendizado é coordenado por um tutor; (iv) o currículo inclui um número limitado de aulas presenciais; (v) a continuidade do processo de aprendizado é diretamente dependente do aluno; (vi) o currículo possui amplo espaço de tempo para estudos autônomos.

Martí et al. (2006) demonstra a eficácia da estratégia educacional PBL em um experimento envolvendo 65 alunos cursando a disciplina de computação gráfica na Universidade Autônoma de Barcelona. Com equipes de 5 pessoas os alunos desenvolveram projetos de forma autônoma, fazendo uso de bibliotecas de manipulação de gráficos sob a supervisão de um professor orientador. Neste experimento foram propostos dois problemas principais: a criação de uma maquete interativa do sistema solar e uma representação tridimensional de um jogo de xadrez. Os autores concluem que PBL é uma boa alternativa para reduzir a carga de trabalho sobre o aprendiz quando comparada a metodologias tradicionais, além de tornar o processo de aprendizado mais dinâmico e menos monótono. No entanto, esta metodologia não pode ser aplicada a qualquer disciplina. Cabe ao professor a tarefa de avaliar a sua viabilidade.

Magalhães e Cavalcante (2011) apresentam uma experiência onde foram avaliadas as opiniões de participantes quanto a efetividade e qualidade, implantada em uma instituição de ensino médio. $\mathrm{O}$ artigo ressalta a importância do treinamento, tendo em vista o mercado de trabalho, como meio de grande importância para a formação do aluno. A pesquisa foi exploratória e qualitativa com escassez de dados estatísticos e com a consulta de somente três alunos do ensino médio. 
Rocha e Araújo (2013) apresentam uma metodologia de projeto para criação e validação de jogos sérios em simulação e treinamento sem estar sujeito aos custos e riscos do processo tradicional. Esta metodologia é formada por quatro etapas: planejamento, produção, execução e avaliação. Os autores destacam bons resultados na integração de equipes multidisciplinares, mas não focam em propostas além dos projetos de treinamento ou em uma metodologia de ensino e desenvolvimento para diferentes jogos sérios com áreas de domínio diversificadas.

Souza e Hack (2014) apresentam uma metodologia de projeto "mídia-educação" como forma de criar jogos. São apresentadas três estratégias de processo metodológico: o uso dos meios, onde mídias digitais são usadas como instrumentos de auxílio a aprendizagem; a leitura crítica dos meios com a análise interpretativa da mídia; e a produção de mídia, que representa o processo de criação e desenvolvimento da mídia. Como resultado é destacado que esta metodologia proporcionou uma aproximação mais completa entre educador e aluno, contudo não destacou no desenvolvimento ou ensino de jogos como fator de incentivo integrado a uma metodologia de ensino específica.

A partir da análise destes trabalhos, decidiu-se aplicar o modelo PBL, uma vez que se mostrou ser um método eficaz quanto a aquisição de conhecimento e por se compatível com os requisitos de trabalho em equipe e autonomia dos participantes.

\section{Metodologia}

O projeto Fábrica de Games tem como objetivo instruir grupos de alunos através de seminários, oficinas e tarefas orientadas por professores, coordenadores técnicos e especialistas em diferentes áreas de atuação usando abordagens inspiradas na aprendizagem ativa.

Norman e Spohrer (1996) afirmam que a utilização da aprendizagem ativa (active learning) incentiva o aluno a procurar por novos conhecimentos para a resolução de problemas. Além disso, proporciona uma atitude mais positiva pelos estudantes, retém o conhecimento por um período de tempo maior, aumenta as habilidades de planejamento, organização e execução (Prince, 2004). Esta metodologia é modelo experimental na qual possibilita aos alunos a oportunidade de participarem na criação e implementação de sistemas com clientes reais para que sejam direcionados às pessoas e as necessidades da comunidade (Hoxmeier, 2003).

Uma ilustração muito utilizada para exemplificar o nível de aprendizado ou conhecimento adquirido no uso desse método de ensino é a pirâmide de aprendizado, representada na Figura 1 (Hadjerrouit, 2015).

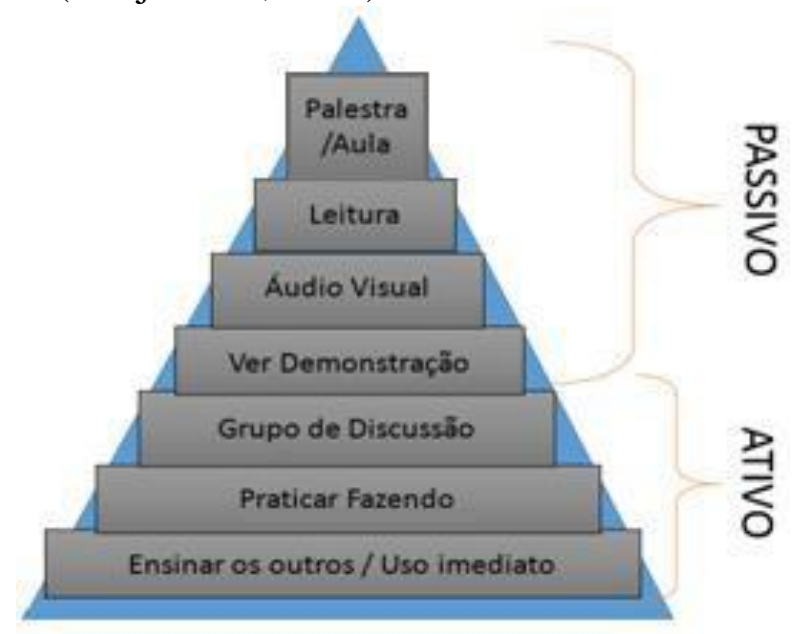


Figura 1: Pirâmide de aprendizado concebida pelo National Training Laboratories of the United States. Adaptado de (Coelho, 2013).

No topo dessa pirâmide observa-se que o nível de conhecimento retido é menor caso se utilize métodos como palestras ou aulas. Este modo de aprendizado é chamado de modo de aprendizagem passiva, visto que o aluno atua como um agregador de conteúdo recebendo as informações sem sua ação direta. Já na base da pirâmide, observa-se que as técnicas como "Praticar fazendo", "Ensinar os outros" e "Uso imediato", possibilita uma maior fixação do aprendizado devido ao interesse autônomo de busca pelo aprendizado por parte dos alunos (Coelho, 2013).

Alguns autores sugerem que as técnicas mais próximas do topo da pirâmide não devem ser desprezadas, ao contrário, podem ser utilizadas como pontos de partida de algum tópico introdutório (Gao e Schwartz, 2015). O projeto Fábrica de Games atua com uma mesclagem das técnicas nas etapas iniciais (aulas e conteúdos audiovisuais) e com características da aprendizagem ativa na etapa intermediária e final do desenvolvimento (reuniões de discussão e prototipação).

No decorrer da Fábrica de Games, os alunos passaram por uma seleção e entrevista a fim de identificar as suas aptidões em relação ao projeto. O objetivo desse passo é fazer um direcionamento prévio para a capacitação em programação ou arte gráfica. Logo optouse por estabelecer dois subgrupos de alunos divididos entre programadores e artistas gráficos, de modo a focar no aprendizado e submeter atividades práticas dessas duas áreas de atuação.

Apesar da divisão dos alunos em dois subgrupos, todos receberam as aulas de programação e arte gráfica, porém os alunos eram direcionados apenas às tarefas do seu grupo pertencente. O presente projeto é dividido em duas grandes etapas: capacitação e prototipação.

\subsection{Capacitação}

A capacitação foi realizada no mês de abril de 2015 e contou com 2 aulas presenciais por semana em torno de 1 hora e 20 minutos. As ferramentas ensinadas foram 0 Unity(Unity3D.com, 2015), Blender(Foundation, 2015) e Gimp(Gimp.org, 2015). As aulas foram ministradas por coordenadores técnicos com experiência nas ferramentas explanadas. Aulas teóricas, exercícios e atividades com questões básicas e intermediárias foram repassadas para os alunos para a fixação do conteúdo.

Entre os conteúdos referentes a área de artes gráficas, destacou-se a modelagem 3D e os processos básicos de criação de modelos tridimensionais para os games como esboços iniciais no papel, blueprints, ferramentas de modelagem 3D e texturização. A criação e edição de texturas e criação de elementos 2D com o manipulador de imagens Gimp também foram explanadas.

Sobre a área de programação foram ministrados conceitos básicos da engine Unity e seu funcionamento. Tarefas práticas com duração de uma semana envolveram os conteúdos expostos em sala como: linguagem de programação $C \#$, funções de física e controle de comportamento do jogo.

\subsection{Prototipação}

A prototipação é utilizada quando os desenvolvedores de um produto de software não possuem a certeza sobre as características dos programas computacionais e desejam entender melhor o que deve ser implementado, quando os requisitos são confusos ou obscuros (Pressman 2006). 
Neste trabalho, o desenvolvimento de protótipos de jogos foi realizado nos meses de maio e junho de 2015. Nesta etapa os alunos são motivados a colocar em prática o que foi submetido durante a capacitação.

Foi proposto para os alunos o desenvolvimento de jogos sérios que possam beneficiar áreas como a educação e saúde. Nesse contexto foi aberto parcerias com outras faculdades para fomentar a integração com áreas diversas, além da computação.

Especialistas foram contatados para auxiliar na criação desses jogos. Dentre os especialistas que contribuíram com o projeto estão: um psicólogo, um odontólogo e um fisioterapeuta. Eles forneceram os requisitos mínimos dos problemas que poderiam ser solucionados com o desenvolvimento de serious games.

Os alunos foram divididos em 3 equipes de 4 alunos cada. No começo da etapa de desenvolvimento dos protótipos os alunos se reuniram com os especialistas e um coordenador técnico. Essa reunião foi necessária para o entendimento do problema fornecido pelo especialista e para a execução de um brainstorm gerando ideias do game a ser desenvolvido.

\section{Jogos desenvolvidos}

Esta seção exibe os protótipos de jogos desenvolvidos pelos alunos após a capacitação. Os jogos foram propostos para auxiliar na solução de problemas em áreas como saúde e educação. A Figura 2 mostra telas dos jogos desenvolvidos.

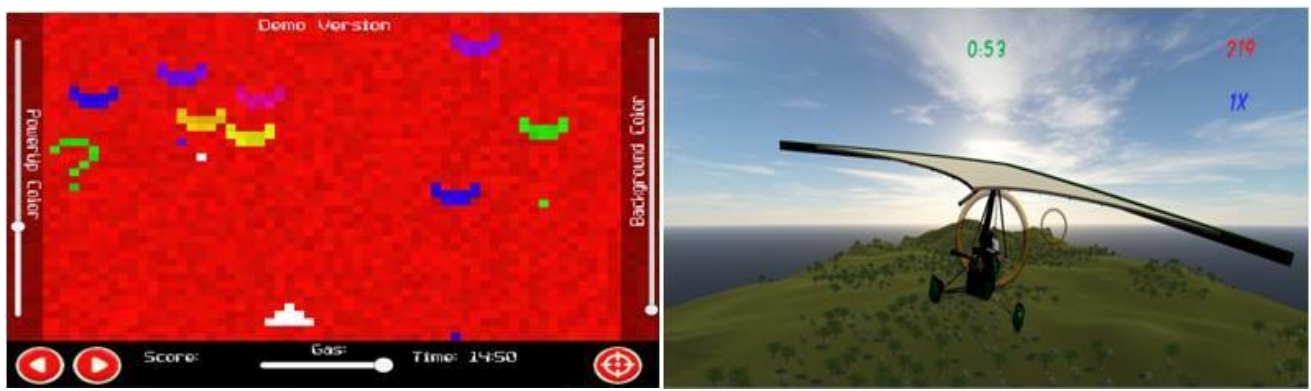

(a)

(b)

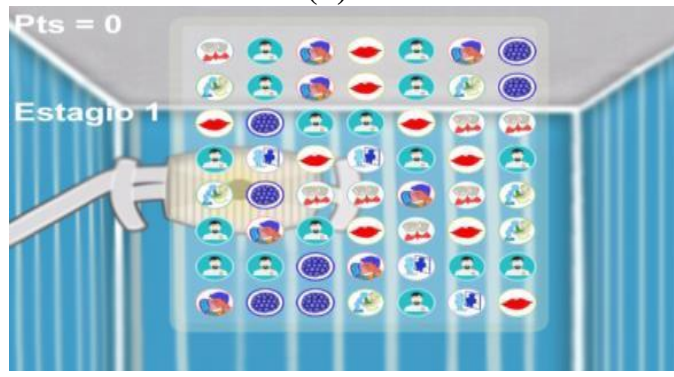

(c)

Figura 2: Gameplay dos jogos prototipados: (a)ChromoNautics, (b)ElderFit, (c)Katzuru.

\subsection{Jogo para identificação de daltonismo}

Discromatopsia ou daltonismo é uma deficiência congênita que geralmente prejudica o reconhecimento das cores verde e vermelho, contudo, existem outros tipos de discromatopsia que alteram a visão para outras cores (Sato et al. 2002). Para ajudar na detecção de diferentes tipos de daltonismos em crianças, os alunos da capacitação desenvolveram o jogo sério Chromo Nautics (Figura 2(a)) baseado no estilo do jogo Space Invaders. Este jogo busca tornar-se uma alternativa viável para os testes de daltonismo conhecidos como o teste CambridgeColour(Goulart, 2009). Desta forma incentiva-se a 


\section{6}

realização dos testes por pessoas que podem não se sentir motivadas com os métodos tradicionais.

O jogador controla uma nave que dispara projéteis contra naves inimigas, pontuando caso ele acerte. A ideia é que alguns itens especiais com cores diferenciadas sejam adicionados no jogo. O jogador deve visualizar e acertar os itens especiais que entram em contraste com a cor do plano de fundo. A comparação do item especial entre o plano de fundo que é confirmado pelo acerto ao item especial, representa o teste de daltonismo.

Para o especialista, esse processo serve para identificar um indício de uma anomalia visual do jogador devido a confusões de cores entre os itens com cores diferenciadas em relação ao plano de fundo. Isso pode ser identificado pelo desperdício do jogador na oportunidade de obter o acerto do tiro no item especial. Esta ideia está sendo aprimorada junto ao especialista.

\subsection{Jogo para o auxiliar no desenvolvimento de equilíbrio de idosos}

Ao atingir uma certa idade as pessoas começam a perder a força em determinados músculos que ajudam a manter o equilíbrio do corpo. Isso gera um risco de quedas que aumenta entre pessoas idosas (Guimarães et al. 2004). Para auxiliar nos exercícios de tratamento para o equilíbrio, o jogo ElderFit (Figura 2(b))foi desenvolvido. O seu objetivo inicial é agregar um conjunto de minigames voltados para idosos que possuem dificuldade de equilíbrio.

Um dos minigames consiste no deslocamento lateral de uma asa-delta conforme a movimentação da cabeça e tronco do usuário. O objetivo do jogador é coletar as argolas flutuantes. Esta movimentação é captada com o dispositivo Kinect(Kinect, 2015) que possibilita a detecção de movimentos dos membros superiores e inferiores. A movimentação da asa-delta é o principal elemento deste jogo, pelo qual os dados referentes ao equilíbrio do idoso serão coletados. Posteriormente, são disponibilizados relatórios com a performance do paciente para os especialistas.

\subsection{Jogo para a educação da higiene bucal}

O terceiro jogo desenvolvido durante a prototipação está voltado para a educação da saúde bucal (Figura 2(c))entre os adolescentes na faixa etária de 13 a 18 anos. Segundo o especialista colaborador desse protótipo, alguns formatos tradicionais de educação como palestras e folders são pouco cativantes ou atraentes para adolescentes. Sendo assim, é proposto a criação de um novo método educacional que possa trazer aos adolescentes uma experiência lúdica e de fácil acesso além de disseminar o conhecimento sobre as doenças bucais.

O jogo é baseado em jogos de sistemas de match 3 bastante conhecidos do mercado mobile como Candy CrushSaga(Candy Crush, 2015). Esses tipos de jogos funcionam com elementos diferentes aleatoriamente distribuídos em um grid, ou malha, onde são eliminados quando 3 elementos iguais são combinados na horizontal ou na vertical.

\section{Resultados}

Esta seção expõe alguns resultados do projeto Fábrica de Games coletados através de questionários, pré e pós-testes. Buscou-se identificar o interesse do aluno em atuar como programador ou artista gráfico, o conhecimento que o aluno considera que possui e o aprendizado do aluno através dos testes.

Um dos resultados está relacionado ao interesse do aluno em ser programador ou artista gráfico. Foram realizadas duas perguntas para os alunos: 


\section{7}

1. Qual seria o seu interesse em ser programador de games?

2. Qual seria o seu interesse em ser artista gráfico de games?

Os alunos responderam às perguntas com valores expressos em porcentagem $(100 \%$ altíssimo interesse, $75 \%$ muito interesse, $50 \%$ médio interesse, $25 \%$ pouco interesse, $0 \%$ nenhum interesse). A Figura 3(a) mostra um gráfico em relação ao interesse do aluno em atuar como programador ou artista gráfico 3D e $2 \mathrm{D}$ com base no teste aplicado antes e depois da capacitação.

Foi observado que anteriormente a capacitação houve um maior interesse dos alunos em ser artista gráfico que posteriormente diminuiu. O interesse em ser programador de jogos aumentou. As causas mais prováveis das variações observadas para o ocorrido pode ser o fato dos alunos já terem conhecimentos em programação e a partir dos conhecimentos adquiridos em artes gráficas 3D e 2D, tenham identificado uma certa complexidade no processo de arte gráfica. Geralmente, o processo de modelagem gráfica, mesmo em ferramentas computacionais avançadas, requer habilidades artísticas práticas com as quais muitos podem não se habituar. Por outro lado, a programação de jogos, abrange desde a codificação como também aspectos gerais dos jogos, como definições de parâmetros, logica matemática, regras e condições de jogo.

Os alunos também apontaram qual seria o seu nível de conhecimento em programação e arte gráfica 3D e 2D responderam às perguntas:

1. Qual seu conhecimento em programação de games?

2. Qual seu conhecimento em artes gráficas para games?

A Figura 3(b) mostra a percepção do nível de conhecimento, a partir dos próprios alunos, em relação ao conhecimento adquirido para as duas áreas abordadas na capacitação. Além disso o gráfico mostra que houve um aumento significativo de conhecimento para as duas áreas.

Interesse por área de atuação

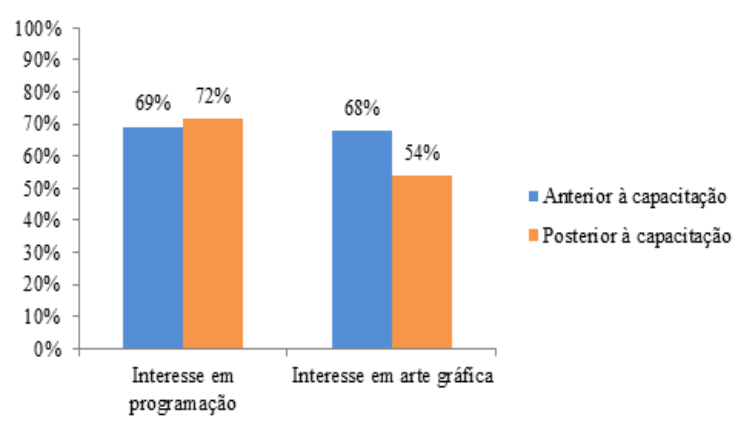

(a)

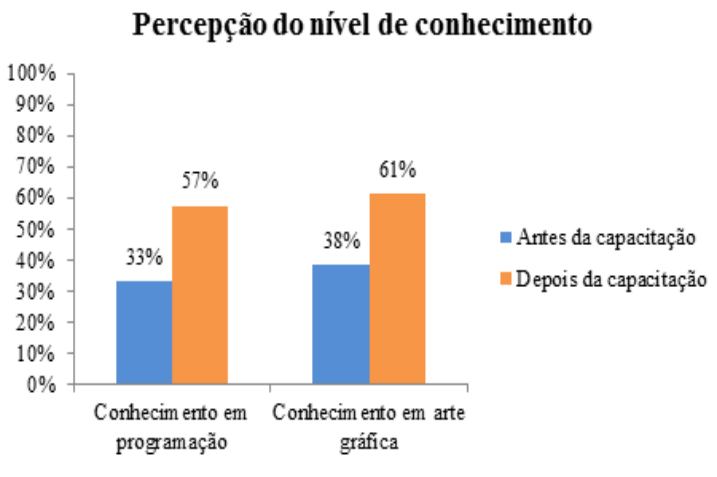

(b)

Figura 3: Gráficos de (a)Interesse e (b) Percepção de Nível de conhecimento

Anteriormente a capacitação, um pré-teste foi aplicado com algumas questões referentes aos tópicos que seriam ministrados. $\mathrm{O}$ teste foi composto por cinco questões relacionadas à programação de jogos e cinco questões para arte gráfica 3D. Após a capacitação e desenvolvimento dos protótipos foi realizado um pós-teste idêntico ao primeiro teste. A partir dos dados foram geradas as taxas de acertos referentes ao grupo de programadores e artistas gráficos. A Figura 4(a) mostra a taxa de acerto para o grupo de programadores e a Figura 4(b) mostra a taxa de acerto com relação ao grupo de artistas gráficos. 


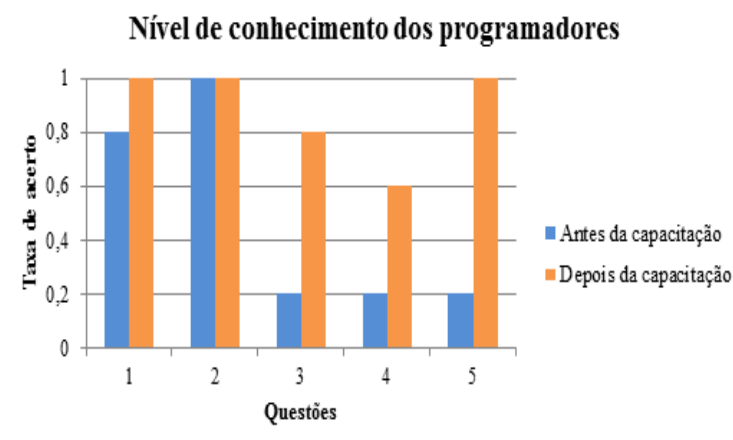

(a)

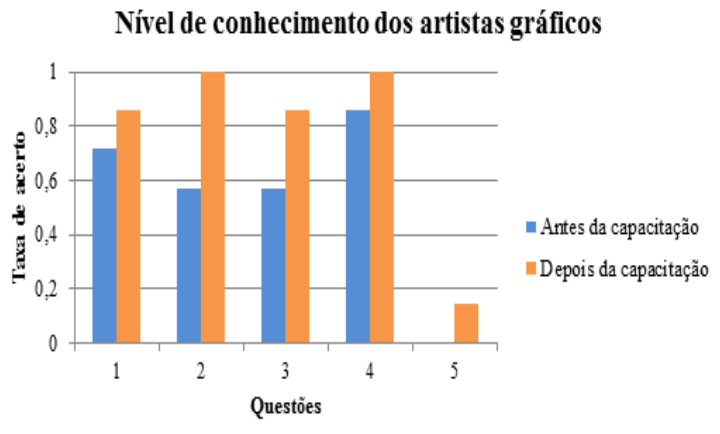

(b)

Figura 4: (a)Nível de conhecimento dos alunos programadores, (b) Nível de conhecimento dos alunos artistas gráficos.

Os dados coletados mostram que houve um aumento no conhecimento após a capacitação. Portanto, os alunos programadores e artistas gráficos tiveram uma melhor taxa de acerto, dos tópicos ministrados, após a execução da capacitação. No entanto, é válido destacar que a abordagem para essa avaliação de conhecimento representa apenas um indício positivo do sucesso da metodologia empregada e é possível que outras variáveis sejam necessárias para melhorar a precisão da avaliação.

No aspecto geral da capacitação, percebeu-se que os alunos se mostraram mais seguros em relação aos tópicos explanados durante a capacitação. Isso foi identificado no decorrer das reuniões para o desenvolvimento dos protótipos. No contexto geral, o conhecimento em programação teve maior consolidação e isso pode ser indicado pelo interesse em atuar como programador de jogos.

\section{Conclusão}

A experiência da estratégia de aprendizagem ativa e atividades implantadas possibilitou uma análise do interesse e aprendizado do aluno e uma perspectiva para o desenvolvimento de jogos sérios. Os alunos se mostraram engajados com a construção dos seus jogos sérios baseado nos problemas apresentados por especialistas de diversas áreas de domínio.

Considera-se que a metodologia, utilizando a aprendizagem ativa como foco, foi útil em dar ao aluno maior interação com outras áreas além da computação. Observou-se a autonomia de alguns membros da equipe em buscar soluções técnicas entre os colegas, pela internet ou pelos materiais disponibilizados. Mesmo com essa autonomia as dúvidas que surgiram foram suplantadas pelos especialistas e instrutores durante as reuniões periódicas do projeto.

O desenvolvimento de projetos reais mostrou-se proveitoso, oferecendo ao aluno o controle de suas tarefas e uma oportunidade de interagir cooperativamente com os colegas de equipe e profissionais de áreas de atuação distintas de modo a produzir novos produtos para o interesse público.

\section{Referências}

CANDY CRUSH SAGA. King.com. Acesso em julho de $2015 . \quad$ Disponível em:<https://king.com/pt_BR/\#!/play/candycrush>.

CARVALO, M. B.; BELLOTI, F.; BERTA, R.; GLORIA A. D.; SEDANO, C. I.; HAUGE, J. B.; HU, J.; RAUTERBERG, M.; An activity theory-based model for serious games analysis and conceptual design. Computer \& Education, V.87 No 2 p. 166-181, 2015.

COELHO, T.;Alunos aprendem melhor com formas de estudo prazerosas. Acesso em julho de 2015. Disponível em: <http://www.usp.br/aun/imprimir.php?id=5202> 
CONNOLLY, T. M.; BOYLE, E.A.; MACARTHUR, E.; HAINEY, T.; BOYLE J.M. A systematic literature review of empirical evidence on computer games and serious games. Computer \& Education, V.59 № 2 p. 661-686, 2012.

FOUNDATION, B.; Home of the Blender project - Free and Open 3D Creation Software. Acesso em julho de 2015. Disponível em: <http://www.blender.org/about/>

GAO, X.; SCHWARTZ, B.; Classroom Implementation of Active Instructional Strategies for Undergraduate STEM Education. International Journal of Information and Education Technology - IJIET, V.5 N 99 p.688-692, 2015. Acesso em julho de 2015. Disponível em:〈http://www.ijiet.org/papers/593-X0003.pdf〉

GIMP.ORG. GIMP - The GNU Image Manipulation Program. Disponível em: <http://www.gimp.org>. Acesso em: 23 jul. 2015 .

GIRARD, C.; ECALLE, J.; MAGNAN, A.; Serious games as new educational tools: how effective are they? A metaanalysis of recent studies. Journal of Computer Assisted Learning. V.29 N³ p.207-219, 2013.

GOULART, P.; Discromatopsias congênitas e condução de veículos. Congresso Brasileiro de prevenção de cegueira e reabilitação visual. 2002. Acesso em julho de 2015. Disponível em: 〈http://www.scielo.br/pdf/abo/v65n1/9574.pdf〉

GUIMARÃES, L.H.C.T.; GALDINO, D.C.A.; MARTINS, F.L.M.; VITORINO, D.F.M.; PEREIRA, K.L.; CARVALHO, E.; Comparação da propensão de quedas entre idosos que praticam atividade física e idosos sedentários. Revista Neurociência. V.12 No2 p.68-72, 2004. Acesso em Julho de 2015. Disponível em: <http://services.epm.br/dneuro/neurociencias/Neurociencias12-2.pdf\#page=11>

HADJERROUIT, S.;Exploring the Effect of Teaching Methods on Students' Learning of School Informatics. Proc. of Informing Science \& IT Education Conference - InSITE. p.201-219, 2015. Acesso em julho de 2015. Disponível em: <http://proceedings.informingscience. org/InSITE2015/InSITE15p201-219Hadjerrouit1510.pdf >.

HOXMEIER, J.; MARIA, M. L.; Service-Learning in Information Systems Courses: Community Projects that Make a Difference. Journal of Information Systems Education. V.14 p. 91 - 100, 2003. Acesso em Julho de 2015. Disponível em:〈http://jise.org/Volume14/14-1/Pdf/14(1)-091.pdf>

KAPP, K.M.; The Gamification of Ledarning and Instruction: game-based methods and strategies for training and education. Editora: Pfeiffer, 2012.

KINECT.Kinect for Windows. Acesso em juho de 2015. Disponível em: <https://www.microsoft.com/enus/kinectforwindows>

MARTí E., GIL, D.; JULIÁ C.;A PBL Experiment in the Teaching of Computer Graphics. Computer Graphics forum. V.25 N $\quad \mathrm{N}^{\mathrm{1}}$ p.95-103, 2006. Acesso em Novembro de 2015 . Disponível em:<http://www.researchgate.net/publication/227760887_A_PBL_Experience_in_the_Teaching_of_Computer_Graphics $>$

NORMAN, D.A; SPOHRER, J.C.; Learner-centered education. Communications of the ACM, V. 39 No $^{\text {4 }}$, p. 24-27, 1996. Acesso em julho de 2015. Disponível em:〈http://itforum.coe.uga.edu/AECT_ITF_PDFS/paper12.pdf>

PRESSMAN, R.; Engenharia de Software. Editora: McGrawHill, 3ed, p.35-38, 2006.

PRINCE, M.; Does active learning work? A review of the research, J. Eng. Educ., V.93 №3, 2004. Acesso em julho de 2015. Disponível em:〈http://www4.ncsu.edu/unity/lockers/users/f/felder/public/Papers/Prince_AL.pdf>

ROCHA, R. V.; ARAUJO, R. B.; Metodologia de Design de Jogos Sérios para Treinamento: ciclo de vida de criação, desenvolvimento e produção. Proc. of XII Simpósio Brasileiro de Jogos e Entretenimento Digital - SBGames 2013, $\begin{array}{llllll}\text { pp.1-10, 2013. Acesso julho em de } 2015 . & \text { Disponível } & \text { em:< }\end{array}$ http://www.sbgames.org/sbgames2013/proceedings/artedesign/09-dt-paper.pdf>

RODRIGUES, H. F.; MACHADO, L. S. E.; VALENÇA, A. M. G.; Definição e Aplicação de um Modelo de Processo para o Desenvolvimento de Serious Games na Área de Saúde. Proc. Congresso da Sociedade Brasileira de Computação - Workshop de Informática Médica, p. 1532-1541, 2010. Acesso em julho de 2015. Disponível em:〈http://www.de.ufpb.br/ labteve/publi/2010_wim2.pdf>

SATO, M. T.; MOREIRA, A. V.; GUERRA, D. R.; CARVALhO, A. C. A; JUNIOR, C. A. M.; Discromatopsias congênitas e condução de veículos. Congresso Brasileioa de prevenção da cegueira e reabilitação visual, September, Curitiba. p. 53-58. 2002 
SAVI, R.; ULBRICHT, V. R.; Jogos Digitais Educacionais: Benefícios e Desafios. Novas Tecnologias na Educação. V. $6 \mathrm{~N}^{\mathrm{o}}$ 2, 2008. Acesso em julho de 2015. Disponível em: <http://www.seer.ufrgs.br/renote/article/viewFile/14405/8310〉. Acesso em: 21 jan. 2015.

SAVI, R.; ULBRICHT, V. R.;Jogos Digitais Educacionais: Benefícios e Desafios. Novas Tecnologias na Educação. V. 6 $\mathrm{N}^{\circ}$ 2, 2008. Acesso em julho de 2015. Disponível em: 〈http://www.seer.ufrgs.br/renote/article/viewFile/14405/8310>. Acesso em: 21 jan. 2015.

SCHMIDT, H. G.; ROTGANS, J. I.; YEW, E. H.; 2011. The process of problem-based learning: what works and why. Medical Education. Medical Education. V.45, No8, p.792-806, 2011.

SOUZA JUNIOR, A. F.; HACK, L.; O PIBID-EF/UNEMAT e a Produção de Jogos Digitais e Não-Digitais: aproximando os nativos digitais e as aulas de educação física. XIII Simpósio Brasileiro de Jogos e Entretenimento Digital - SBGames 2014. p. 660-662, 2014. Acesso em julho de 2015. Disponível em:<http://www.sbgames.org/sbgames2014/files/papers/culture/short/Cult_Short_O\%20PIBID-EF-

UNEMAT\%20e\%20a\%20Producao\%20de\%20Jogos.pdf>

UNITY3D.COM.Unity - Overview. Acesso em julho de 2015. Disponível em: 〈https://unity3d.com/pt/unity>

VILELA, R.; BORGES, R.; Metodologia de Design de Jogos Sérios para Treinamento: Ciclo de vida de criação, desenvolvimento e produção. XII Simpósio Brasileiro de Jogos e Entretenimento Digita -SBGames 2013. p.63-72, 2013. Acesso em julho de 2015. Disponível em: <http://www.sbgames.org/sbgames2013/proceedings/artedesign/09-dtpaper.pdf>. 